\title{
Research on landscape design of community pension under the background of aging
}

\author{
Tian $\mathrm{Wei}^{1, \mathrm{a}}$, Zhigang $\mathrm{Li}^{1, \mathrm{~b}}{ }^{*}$ \\ ${ }^{1}$ College of art and design, Wuhan institute of technology, Wuhan, China
}

\begin{abstract}
As a country with a large population in the world, China is currently aging. This paper starts from the basic needs of the elderly's care environment, and takes the elderly's psychological needs, physiological needs and behavioral needs as the starting point, and explores the ecological design of community landscape under the background of aging to meet the many needs of today's aging population.
\end{abstract}

\section{INTRODUCTION}

China has the largest aging population in the world. Internationally, standard for a country or region of aging is the population over 60 years old accounts for $10 \%$ of the total population, or the population over 65 years old accounts for 7\% of the total population.[1] In China, the proportion of people over 65 is $13 \%$. China has entered an aging society. In this context of aging, it is necessary to optimize China's pension model from the perspective of community pension space landscape design, so as to improve China's pension system. Pension model of China has always based on the family, which is also a traditional virtue of the Chinese nation. However, considering the background of the rapid growth of population aging, it has become challenges for landscape designers to design the landscape of community pension space to provide a suitable and healthy community pension environment for the elderly.

\section{CuRRENT SITUATION AND PROBLEMS}

\subsection{Lack of physiological demand for facilities and transportation under aging}

At present, it is a common phenomenon that residential areas are designed around circular roads and many motorways. The interior of the community is mainly pedestrian roads. However, in the old residential area, The erderly can walk only on roadway due to parking chaos and footpath occupation, especially in the peak period of students' going to school, traffic chaos, can not guarantee the safety of the elderly travel.

Today, the elderly in many family communities have to use crutches and wheelchairs which are inconvenient for outdoor activities, and they are not able to move. However, the layout of the sidewalk rarely consider the decline of the physical function of the elderly, there are no seats and supporting devices for rest in community layout. At the drop of the road, the steps are utilized, which leads to the reduction of comfort and risk of walking.

Through the current community landscape design, we can find that the traffic is chaotic and the safety of outdoor activities can not be guaranteed. Appropriate road space is a prerequisite for the elderly to walk outside safely. At present, It is traffic chaos and the mix of people and vehicles that bring serious trouble to the elderly travel. The problem of sidewalk occupation in old communities is more serious. Due to the limited number of parking spaces, many private cars have to occupy the narrow sidewalk or public space, which leads to more crowded roads, limits the range of activities, and increases the possibility of mixing pedestrians and vehicles to a certain extent, which is not conducive to the activities of the elderly and raises the risk. Especially in the road intersection or the node location with large traffic flow, there is a lack of corresponding signs such as deceleration and stop prohibition. Because the elderly's response and action are slow, they can't react effectively. So limiting the speed of the vehicle gives both the old people and the driver plenty of time to react, which is of great significance to avoid accidents.

Due to the degradation of the elderly's physical function, we should consider their physiological needs when designing the landscape of community pension space. Ensuring enough sunlight is beneficial to the health of the elderly, but too much sunlight will also cause harm to the elderly. Therefore, the allocation of shading facilities must be seriously considered. Moreover, lighting devices must also take into account the deterioration of vision of the elderly. In the design of night lighting, the reasonable integration into the environment should be focused on. Moreover, in the landscape design of community pension space, the placement of all kinds of guidance and warning signs must be greatly taken into consideration. Because of poor eyesight and short memory of the elderly, the guidance 
and warning signs should be easy to identify, which is convenient for the elderly to recognize from a distance.

In the design of walking path, we should also refer to the living habits of the elderly to meet the needs of anti-skid and barrier free path. Especially in the pedestrian crossing or intersection with large flow of people, there are not enough signs of deceleration and parking. Due to the slow reaction of the elderly, they will not be able to respond immediately and effectively in case of danger. Enhance, based on this assumption, limiting the speed of the vehicle will allow not only the elderly, but also the driver to respond to a specific time. It is very important to avoid accidents.

\subsection{The behavior demand of lack of activity space under aging}

Usually, the elderly need to take care of their kids, when the children play in the fitness area. In the meanwhile, the elderly also have their own entertainment. For instance, some chat with others, some play chess and cards in the square. In order to meet the need of recreational activities, this kind of permeable space design is worth promoting. A variety of design concept can be proposed for landscape functions to meet elderly requirements based on their daily routines.

The area where the elderly aggregate spontaneously is mainly used for leisure and entertainment. Because this kind of space is generated by people's spontaneous gathering, there is not enough infrastructure in the space, such as tables and chairs, which are prepared by the elderly themselves. There is no obvious separation between the quiet rest area and the dynamic space, which makes the division of dynamic and static areas unreasonable and causes mutual interference.

As far as the level of landscape space is concerned, many old communities are not rich enough, while a small number of communities even do not. Most of the landscape space of the old communities in China is based on evergreen plants. For example, camphor trees are mainly planted in Southwest China. The color change is not strong, and there is no aesthetic feeling, and there is no interaction with the elderly. Although the fitness area is equipped with Chinese parasol, osmanthus fragrans Lour, palm trees and other vegetation, there are not many species and species, and the distinction of landscape between four seasons is not obvious. The place lacks storage space, and many of the elderly's personal belongings are hung on branches or on the ground, which brings inconvenience and hinders the view. The lack of lighting or sign is detrimental to the safety of the elderly. However, it is certain that some communities have set up public toilets to meet the urgent needs of the elderly for using toilets during sports.

The performance of physical aging of the elderly has a great impact on the demand for outdoor activities, primarily including illumination, acoustic environment, thermal environment and barrier free design. [2] The elderly has a variety of outdoor activities to participate, so there should be different types of area. In the actual research, it is found that the community only provides large areas without specific space separation, so there is no separation in the same space. Recreation space is rarely considered in most community landscape design. In this case, it is likely to cause the problems of dynamic and static confusion, activity disorder and mutual influence. In the design, low shrubs or landscape sketches can be used for proper isolation, which not only have broader visual range, but also promotes mutual communication. It can also increase independent activity space.

At present, the community is dominated by evergreen plants. It can provide green space for the community all year round and seems full of vitality. However, with few ornamental effect, a wide range of evergreen plants lack interest and beauty. The dense greenery easily blocks out the sun, leading to a lack of sunlight in winter, which also reduces utilization of space. And these plants can hardly interact with people. Generally, most of the plants in the community are green leafy vegetation with few or no flowers. In the configuration of plants, it is necessary to select and add varieties according to the characteristics of plants. It is necessary to select non-toxic, thornless and non- irritating plants. For example, the most common ones are Honeysuckle, sweet scented Osmanthus. However, some plants like willow are not suitable for planting in the community, because catkins are likely to cause skin allergy and even respiratory tract infection, which is harmful to the elderly with weak respiratory system.

Due to the differences in behavioral needs, the elderly has different functional space to meet their own activities. Some are used to being alone, some like group activities, some like art and sports. If we want to meet the needs of all kinds of behavior of the elderly, the landscape space of community pension must meet the coexistence of individual and group activity space. The design of single person activity space must ensure privacy and security function to create a relaxed and shady environment. Collective activity space is proposed for outdoor activities, such as outdoor dance, fitness and other activities, to promote the interpersonal communication of the elderly.

Moreover, many old people have the behavior demand of planting plants. In the face of this situation, we can arrange a piece of private land for the elderly to grow on their own. At the same time, the horticultural therapy in the concept of foreign health care can be integrated into it. Horticultural therapy was initially used in hospitals and pension communities to enable the elderly and patients to participate in the landscape, which promote physical activities, strengthen the body, and cultivate the psychological satisfaction of harvest to achieve mental health. Integrating this concept into the landscape space of community pension can also promote the physical and mental health of the elderly and enrich their life time. This approach will meet the behavioral needs of the elderly. At the same time, it is also a sustainable green development model. 


\subsection{Lack of the psychological needs of safety and culture in the aging society}

Aging is an inevitable stage for people. As a special and huge elderly group in the current society, the psychological problems are characterized by aging, universality, epochal and diversity. And it is multiple factors like Physiological changes, social development, family relations and individual living habits of the elderly that lead to various psychological changes of the elderly. [3] At this stage, the physical strength and thinking ability of the elderly continue to decline, and the elderly can hardly control their own behavior very well. Therefore, in the living environment of the elderly, it is important to provide infrastructure to ensure security for the elderly, which is also one of the psychological needs of the elderly.

Satisfying the regional culture is also one of the psychological needs of the elderly. The elderly has rich experience and special feelings for their own living area. Chinese people always have the idea of returning to their roots, which fully reflects the deep feelings of the elderly about their hometown and living environment. The familiar environment and culture can make the elderly have a sense of belonging and identity. Chinese people are nostalgic. In the past years when the contemporary elderly grew up, the living buildings and landscapes all have their own strong cultural brand. Each space has distinct regional cultural elements, so in the design of community pension landscape space, we should meet the psychological needs of regional culture.

\section{DISCUSSION}

\subsection{Improving traffic safety of landscape design}

Due to the decline of physical fitness, the elderly are not as flexible as the young in their daily activities. When the elderly are transferred from different places, the decline of reaction ability brings some security risks. The landscape design of the pension space should also fully consider the physiological needs of the elderly, and pay attention to the safety of the passage between different places of the space. For example, in the design of retirement Road, we should avoid long and vertical route planning as far as possible, and adopt diversified road design as far as possible. Combined with the walking characteristics of the elderly, we should establish a safe walking system, wheelchair and other roads. In addition, if there is a slope, the length of the slope shall not exceed $10 \mathrm{~m}$, the longitudinal slope shall not exceed $1 / 12$, and the width shall not be less than $1.2 \mathrm{~m}$, regardless of whether there are steps. Firstly, wheelchair access should be provided. Secondly, safety facilities such as handrails should be designed on both sides of the ramp. The decline of physiological function of the elderly also damages their visual and memory functions. When designing a space for the aged, we also need to fully consider how signs attract the attention and understanding of the elderly. The design should use larger font as far as possible, use color matching with strong contrast and no harm to eyesight, and place warning signs where the elderly can easily see them.

Safety is the basic principle of community endowment. For one thing, the elderly belong to a particularly vulnerable group. Due to their poor physical condition, their abilities in various aspects have declined greatly, which increases the possibility of danger and reduces the recovery degree of injury. For another, the elderly themselves are also vulnerable to insecurity. They want family and social attention. At the same time, they also hope that the environment they live in can provide them with a sense of spiritual security. Therefore, the design of quiet communication space environment, privacy, semi privacy and open space environment can provide them with a sense of security. In addition, other landscape facilities, such as seats, fitness equipment, and other landscape elements, such as Garden Road, ramp, pavement, should also meet the safety principle.

\subsection{Enhance the function of landscape design}

The function compensation of landscape design mainly refers to the space function design of pension space, which needs to make up for the decline of various abilities of the elderly, and effectively support the elderly's independent living ability, and even play the role of health protection for the elderly's weak body. After retirement, due to the lack of social activities and the liberation from the busy pace of life, the physiological function of the elderly is declining more and more quickly. According to the behavior needs of the elderly, in the landscape design, we should consider how to make up for the physical shortage of the elderly, and how to add activities and communication space in the pension space to meet the needs of the elderly. The elderly participate in social activities. For example, in the design of pension space, open space is suitable for the design of leisure area, more suitable for the elderly to rest and talk. In addition, the elderly care space should also be combined with the characteristics of human community, design a special activity center for the elderly group, and develop a broad geographical planning, in order to provide physical and performance space for the elderly care. To meet the behavior and communication needs of the elderly and realize diversified entertainment.

In addition, some elderly people have their own hobby of planting plant landscape, which can be integrated into the landscape design of elderly care with the concept of health care. Such as horticulture therapy under the concept of health care, it is in the whole process that the participants actively participate in the planting of vegetables and fruits, and experience weeding, planting, applying, watering and harvesting. On the one hand, it makes the participants accept physical exercise and thinking training, on the other hand, it makes the participants get a great sense of satisfaction during the harvest period, so as to promote the physical, social and emotional development. Compared with other methods, its biggest advantage is its active participation. 
At the same time, horticultural therapy is not limited to the elderly, they can be the elderly, the disabled, people with intellectual problems, people with mental illness, etc., so that people from all levels of the community can participate. Its contents include handicraft activities, such as flower folding, flower and leaf collage, outdoor games, outdoor courses and other group activities, as well as indoor and outdoor activities such as farming and gardening, flower arrangement, plant planting and nursery. Various effects can be achieved by participating in various horticultural therapy activities. Plant cultivation activities such as sowing, transplanting seedlings, watering and weeding can promote the metabolism of human body and maintain the health of human body; grow together with the planted plants and experience the changes of life, which can enhance people's sense of time and respect for natural life. Through contact with plants, observation, tasting, and selling the fruits of their own labor, they can stimulate the development of five senses, relieve physical and mental fatigue, and obtain a great sense of self satisfaction and achievement. [4] So that the elderly to achieve dual behavioral and psychological satisfaction, and promote the healthy development of the elderly.

\subsection{Pay attention to the regional culture of landscape design}

The psychological needs of the elderly are mainly reflected in their daily behavior, to meet their psychological needs, to give people a natural sense of satisfaction and security. Meeting the psychological needs of the elderly plays an important role in promoting the harmonious development of social environment. In the concept of landscape design of pension space, we should try to understand the user's psychology, have a general understanding of the user's basic information, and pay attention to the cultural connotation in the landscape design, which can meet the psychological needs of the elderly with the support of the rich life experience of the elderly. Elderly care space is an important residence for the elderly in their old age, and Chinese people have the complex of returning to their roots and nostalgia since ancient times. It is very important to deeply understand the local culture for the establishment of the sense of belonging and identity of the elderly. Therefore, the elderly need several kinds of situations to stimulate the participation of the elderly and help them realize different social values from young adults within their ability.[5]

Community pension should reflect the local history and culture. As the old people are deeply impressed by the old memories and tend to be nostalgic, the elements with local historical characteristics will more arouse their emotions, recall the old memories, and produce a continuity in the present and future memories, enhance their sense of identity, and play a role in the mental and psychological rehabilitation and treatment of the elderly. In the current situation of the intensification of urbanization, there is a situation of urban homogenization, that is, the lack of use of local culture, and only when the elderly live at that time, do their own regional cultural elements into the building and landscape space. Nowadays, we need to protect the local ancient buildings and villages, explore deep historical elements or plant local characteristic plants to better reflect the local characteristics of the community, and play a role in the spiritual and psychological rehabilitation and maintenance of the elderly.

In addition, because most of today's society is taken care of by the elderly, so sometimes the children's activity area will be in the activity space of the elderly. With the integration of regional cultural elements into landscape design, children will be influenced by the regional culture of their residence from childhood, so that children can inherit the regional culture and even improve their cultural confidence, rather than being homogenized by the characteristics of modern development.

\section{CONCLUSION}

In recent years, China has fully entered the development stage of aging. The aging population is gradually expanding, and the demand for pension space is also growing year by year. Feasible design of the community landscape can effectively meet the physiological, behavioral and psychological needs of the elderly. Enhance, daily routine of the aged should be prudently considered in the present landscape design, which is supposed to fully understand the needs of the elderly and focus on details like the site landscape greening, safety facilities and activity space, so as to meet the needs of the elderly on the landscape design of community pension space under the background of aging.

\section{REFERENCES}

1. Li Wenyi. An Empirical Study on the impact of population aging on the income and expenditure gap of China's basic endowment insurance fund [D]. Chongqing University, 2018

2. Li Weiping. Research on outdoor activity space design strategy of Nanchang old community based on community pension mode [D]. Nanchang University, 2020

3. Xiao Yuting. Study on landscape design of outdoor activity space suitable for aging in old communities of Nanchang [D]. Jiangxi University of Finance and economics, 2020

4. Guo Changshong. Research on landscape design of elderly community under the concept of rehabilitation Landscape [D]. Changchun University of technology, 2019

5. Chen Yuqian. A preliminary study on the design of a new type of community situational environment for the aged: a case study of Hangzhou Xiasha Jiangwan community environment for the aged [J]. Design, 2015 (11): 142-144 